\title{
Evaluation of factors associated with medication adherence and hypertension among diabetic patients in Pakistan
}

\author{
Ali Hassan-Gillani ${ }^{1,2,3 *}$, Amna Saeed ${ }^{1,2,3}$, Farhat Khanum ${ }^{4}$, Muhammad Q. Uz-Zaman ${ }^{5}$, and \\ Jamshaid Akbar ${ }^{6}$ \\ ${ }^{1}$ Department of Pharmacy Administration and Clinical Pharmacy, School of Pharmacy Xi'an Jiaotong University, Shaanxi, China; ${ }^{2}$ Center for Drug \\ Safety and Policy Research, Xian Jiaotong University, Xi'an, Shaanxi, China; ${ }^{3}$ Shaanxi Centre for Health Reform and Development Research, Xi'an, \\ Shaanxi, China; ${ }^{4}$ Department of Pharmacy, Yusra Institute of Pharmaceutical Sciences Islamabad, Pakistan; ${ }^{5}$ Department of Pharmacy and Alternative \\ Medicine, Islamia University Bahawalpur, Pakistan; ${ }^{6}$ Department of Pharmaceutical Sciences Superior University Lahore, Pakistan
}

\begin{abstract}
Introduction: Non-adherence to a drug regimen is an important issue for the management of diabetes, and it leads to therapeutic failure. Uncontrolled diabetes is a prime reason for other complications, specifically hypertension (HTN). Thus, we evaluated the factors associated with therapeutic adherence to diabetes medication and factors associated with HTN among a population with diabetes in Punjab, Pakistan. Materials and methods: A descriptive cross-sectional study was conducted from December 2015 to March 2016. Systemic sampling technique was adopted to recruit patients. The eight-item Morisky Medication Adherence Scale-8 was used to evaluate adherence. Descriptive statistics were used to elaborate on the variables, bivariate, and multivariable analyses. These were conducted to classify factors independently associated with moderate-high adherence to medication therapy and factors associated with HTN. Results: A total of 299 people completed the study, with a mean age \pm standard deviation, $48.8 \pm 14.6$ years. One hundred and sixty-nine (56.5\%) participants were men, 270 (90.3\%) were married. High adherence was found in 85 (28.4\%), moderate in 69 (23.1\%), and low in 145 (48.5\%) patients. Patients who were taking help from the family members in taking medicine correctly and patients who believe that drug therapy caused side effects were showing reduced moderate-high adherence (adjusted odds ratio [AOR] $=0.562 ; 95 \%$ confidence interval $[\mathrm{Cl}]=0.328,0.961)$ and $(A O R=0.349 ; 95 \% \mathrm{Cl}=0.192,0.635)$, respectively. $A$ total of $44.4 \%$ of patients had HTN as a comorbidity. Family history of HTN $(A O R=23.42 ; 95 \% \mathrm{Cl}=9.655,56.853 ; p<0.001)$, and abnormal cholesterol levels $(A O R=23.401 ; 95 \% \mathrm{Cl}=10.195,53.765 ; p=0.01)$ were significantly associated with a hypertensive status of diabetic patients in our population. Conclusions: Patients who were depending on family members to take medication correctly at a proper time, and the patients who believed that drugs caused side effects were showing a reduced level of adherence to our study group. The risk factors of HTN (i.e., abnormal cholesterol levels) were prevalent among the patients of diabetes; these must be monitored and controlled.
\end{abstract}

Key words: Diabetes. Compliance. Hypertension. Risk factors.

Correspondence:

*Ali Hassan-Gillani

E-mail: hassangillaniali@yahoo.com
Date of reception: 11-10-2019

Date of acceptance: 12-12-2019

DOI: 10.24875/RMU.19000130
Available online: 17-03-2020

Medicina Universitaria. 2020;22(1):27-37 www.medicinauniversitaria.org 1665-5796/@ 2019 Universidad Autónoma de Nuevo León. Published by Permanyer. This is an open access article under the CC BY-NC-ND license (http://creativecommons.org/licenses/by-nc-nd/4.0/). 


\section{Introduction}

Diabetes mellitus (DM) is one of the most common non-communicable diseases. In recent years, DM has been escalating at a drastic rate and has become a global threat ${ }^{1}$. The prevalence of DM has been found to be $6.9 \%$ in Pakistan ${ }^{2}$. This surge in DM prevalence is attributable to urbanization and socioeconomic development, which ultimately alter traditional lifestyles ${ }^{3}$. To achieve desired therapeutic outcomes and disease management, patients must comply with drug therapy. The World Health Organization (WHO) defines compliance as "the extent to which a person's behavior (taking medication, following a diet and/or executing lifestyle changes) corresponds with agreed recommendations from a health-care provider" ${ }^{\prime 4}, 5$. Lower adherence level to prescribed medication is the reason for $30-50 \%$ treatment failure and, in result, leads to organ failure ${ }^{6}$. The prevalence of hypertension (HTN) among people with diabetes is relatively high (ranging from $32 \%$ to $82 \%$ ) compared with people who do not have diabetes. Risk factors for the development of HTN in the general population include older age, male sex, sedentary lifestyle, smoking, abnormal body mass index (BMI), family history of HTN, and dyslipidemia?. The presence of type 2 diabetes, duration of $\mathrm{DM}$, and poor control is also factors that contribute to the development of $\mathrm{HTN}^{7-9}$.

Comprehensive diabetes care and management depend on effectual diabetes education, enhanced knowledge, attitudes, and skills that eventually lead to better control of the disease ${ }^{10}$. The burden on health-care systems can be reduced by intervening to motivate and guide the patient in the right direction ${ }^{11}$. Several factors can hamper adherence, which can be classified as the patient, therapy, health-care system, socioeconomic, and disease-related factors; accessibility falls into the category of health-care facility-related factors ${ }^{12}$. Furthermore, the previous studies showed that other factors, such as improper scheduled time and low patient education, increased medication non-adherence. Furthermore, medication adherence has been influenced by local culture and religious affiliations that influence individual medication behavior ${ }^{13,14}$. Despite giving due recognition to DM as one of the most important health concerns in the middle and old aged, it demands the gathering of related information on medication-taking patterns and factors associated thereof. Although a study was performed by Nazir et al., which evaluated the relationship between medication adherence and glycated hemoglobin (HbA1c) level ${ }^{15}$, the studies on factors affecting medication adherence are very rare in Pakistan. This study was designed to assess the adherence to medication therapy, confounding factors that affect adherence and the prevalence of predictors of HTN status among patients with DM in southern Punjab, Pakistan.

\section{Materials and Methods}

\section{Study design and site}

A descriptive and cross-sectional study was conducted among patients with DM from December 2015 to March 2016. The study site was southern Punjab, Pakistan. The target districts were Bahawalpur, Multan, and Rahim Yar Khan.

\section{Study population and study setting}

All those had been diagnosed as diabetics according to the measuring standards of American Diabetes Association (taking the diabetes medication for at least or more than a year) on oral hypoglycemic or insulin therapy; of any age, sex, religion, and socioeconomic status; and willing to participate in the study. Both type 1 and type 2 patients were captivated. The participant's name and contact or any other personnel information were not collected. Patients who had been diagnosed with DM within the last year, and those who were unwilling or unable to participate (mentally compromised, and unconscious) were excluded from the study. The diabetes treatment is accessed by medical records of hospitalized patients and prescription records of outpatients, and further asking patients if they are taking any additional medication for diabetes. Among all Primary Health Care Centers (PHCC) and private clinics in the study districts, three PHCC and three private clinics were selected. We chose those clinics that included diabetes management and family practice/general medicine/internal medicine and those with a higher patient volume.

\section{Study instrument}

The study instrument consisted of descriptive as well as dichotomous and multiple-choice questions. The descriptive portion queried patient demographics, comorbidities, laboratory test results, and drug therapy; the quantitative portion consisted of dichotomous and multiple-choice questions. Other study variables included possible reasons for non-adherence, such as type 
of medication therapy, affordability, poly-pharmacy, use of a glucometer (GM), and help with medication from family members, accessibility, beliefs about side effects of drug therapy, and beliefs about the benefits of drug therapy. The comorbidities were assessed by the description in the medication charts of the inpatients and prescriptions of outpatients. At least 30 min of walking 5 days a week was considered to be the minimum parameter for exercise compliance or physical activity ${ }^{16,17}$. Any patient who reported cigarette smoking of any kind and in any quantity during the past 12 months was considered a current smoker $^{17}$. Questions were closed-ended in yes/no format. An eight-item Morisky Medication Adherence Scale (MMAS)-8 is the tool widely used for the assessment of adherence to different conditions ${ }^{18-20}$. The Urdu version of the MMAS-8 scale was available, which was translated and validated by Saleem et al. in the study ${ }^{21}$. The rest of the questionnaire was designed in English and translated into Urdu. To ensure that the original meaning of the questionnaire items was maintained, the Urdu version was translated back into English by a researcher acquainted with both languages. The Urdu version of the questionnaire was pretested on 30 diabetic patients outside of the study area, and minor changes were made as needed.

Adherence with medication (both oral medication and insulin) was assessed by the MMAS-8. Responses were summed, with the total possible MMAS-8 score ranging from 0 to 8 . Adherence was high if the mean score was 8 , moderate if the mean score was $\geq 6-<8$, and low if the score was $<6^{22}$. Dichotomization was done based on the given criteria. Patients with score $>6$ were characterized as moderate-high adherent and all others as low-adherent ${ }^{23}$.

To evaluate participants' HTN status, the researchers measured each patient's blood pressure using a standard mercury sphygmomanometer. According to the WHO criteria, patients were considered to have HTN with systolic blood pressure $160 \mathrm{mmHg}$ or more and/or diastolic blood pressure $95 \mathrm{mmHg}$ or more, or if under ongoing treatment with antihypertensive drugs. BMI was calculated using a standard formula (weight in $\mathrm{kg}$, divided by the square of the height in $\mathrm{m}^{2}$ ). $\mathrm{BMI}<18.5$ is classified as underweight, 18.5-24.9 as normal, 2529.9 as overweight (pre obesity), and $\geq 30.0$ is considered obese ${ }^{24}$. Lipid profile (LP) and blood glucose level (BGL) were assessed by testing participant blood samples. Officially recommended by ADA, the normal BGL for a normal person is $140 \mathrm{mg} / \mathrm{dl}$ and for diabetic, its $<180 \mathrm{mg} / \mathrm{dl}^{25}$. The capillary tube whole blood method was used, with a cholesterol lipid analyzer to measure LP. Dyslipidemia was considered present with total cholesterol $>5.60 \mathrm{mmol} / \mathrm{L}$.

\section{Training of data collectors and quality management of data}

Six data collectors were designated to collect data simultaneously in six different territories. The training involved the following aspects: (1) presenting a brief introduction of the study purpose to patients; (2) conducting face-to-face patient interviews; and (3) coping with patient lack of cooperation or other difficulties during the interview. The training was carried out for 3 days, with a demonstration given by the prime researcher. Trainees then conducted a pilot study in each of their respective districts and were observed for their interviewing skills.

\section{Sample size}

The sample size was calculated based on the prevalence of the disease. Assuming the prevalence of disease as $6.9 \%$ in Pakistan ${ }^{2}, 3 \%$ as precision rate, and $95 \%$ confidence interval $(\mathrm{Cl})$, a total of 282 sample size was calculated.

\section{Study sampling}

No diabetes registry or computerized database of DM patients was available in the study districts; therefore, systematic random sampling in the study settings (centers and clinics) was used to select potential participants in the study. Patients attending the private clinics were listed before visiting physicians. The list contains the medical complaints to the attending physician, diabetes status and some other variables. Every third DM patient who was listed as diabetic and visited any of the participating centers and clinics was chosen; a total of almost 900 individuals were approached from hospitals and private clinics. Only 398 of the total responded, of whom 299 completed the study. Three hundred and seventy-three approached patients were unwilling to participate (due to either time limitations or disinterest in the study); 129 patients were mentally compromised or seriously ill and excluded from the study.

\section{Data analysis}

Questionnaires with responses for more than $95 \%$ of questions were considered well completed and were 
Table 1. The demographics of respondents

\begin{tabular}{|c|c|}
\hline Demographic characteristics & Respondents n (\%) \\
\hline $\begin{array}{l}\text { Age }(48.8 \pm 14.6) \\
<25 \\
26-40 \\
41-55 \\
\text { More than } 55\end{array}$ & $\begin{array}{c}17(5.7) \\
71(23.7) \\
116(38.7) \\
95(31.8)\end{array}$ \\
\hline $\begin{array}{l}\text { Locality } \\
\text { Urban } \\
\text { Rural }\end{array}$ & $\begin{array}{l}196(65.5) \\
103(34.5)\end{array}$ \\
\hline $\begin{array}{l}\text { Gender } \\
\text { Male } \\
\text { Female }\end{array}$ & $\begin{array}{l}169(56.5) \\
130(43.5)\end{array}$ \\
\hline $\begin{array}{l}\text { Monthly income (PKR) } \\
<15,000 \\
15,000-30,000 \\
\text { More than } 30,000\end{array}$ & $\begin{array}{c}68(22.7) \\
134(44.8) \\
97(32.4)\end{array}$ \\
\hline $\begin{array}{l}\text { Marital status } \\
\text { Married } \\
\text { Single }\end{array}$ & $\begin{array}{c}270(90.3) \\
29(9.7)\end{array}$ \\
\hline $\begin{array}{l}\text { Education } \\
\text { Less than primary } \\
\text { Primary-Middle } \\
\text { Matric } \\
\text { Graduate }\end{array}$ & $\begin{array}{l}124(41.5) \\
65(21.7) \\
71(23.7) \\
39(13.1)\end{array}$ \\
\hline $\begin{array}{l}\text { Family history of diabetes } \\
\text { Positive } \\
\text { Negative }\end{array}$ & $\begin{array}{c}208(69.6) \\
91(30.4)\end{array}$ \\
\hline $\begin{array}{l}\text { Family history of hypertension } \\
\text { Positive } \\
\text { Negative }\end{array}$ & $\begin{array}{c}97(32.4) \\
202(67.6)\end{array}$ \\
\hline
\end{tabular}

PKR: Pakistani rupee

included in the statistical analysis. Data were entered into EpiData 3.1.0 software and double-checked by the researchers. SPSS for Windows, Version 18.0 (SPSS Inc., Chicago, IL, USA) was used for data analysis. Demographic characteristics were illustrated using descriptive analysis. Percentages were used for categorical variables whereas continuous variables were expressed by mean \pm standard deviation. Relationships between the proposed predictors and moderate-high adherence with DM medication therapy and that between predictors and HTN were assessed using logistic regression. To determine variables independently associated with medication adherence and HTN, variables with $p \leq 0.25$ in the bivariate analysis were selected for multivariable logistic regression. We base this on the Wald test from logistic regression and $p$ value cutoff point of 0.25 . More traditional levels such as 0.05 can fail in identifying variables known to be important ${ }^{26,27}$. The statistical significance of associations among the
Table 2. Clinical characteristics of DM patients

\begin{tabular}{|c|c|}
\hline Variable & n (\%) \\
\hline $\begin{array}{l}\text { Duration of diabetes (years) }(9.36 \pm 6.9) \\
1-5 \\
6-10 \\
11-15 \\
\text { More than } 15\end{array}$ & $\begin{array}{l}100(33.4) \\
109(36.5) \\
47(15.7) \\
43(14.4)\end{array}$ \\
\hline $\begin{array}{l}\text { Smoker } \\
\text { Yes } \\
\text { No }\end{array}$ & $\begin{array}{c}46(15.4) \\
253(84.6)\end{array}$ \\
\hline $\begin{array}{l}\text { Total cholesterol } \\
\text { High (>5.60 mmol/L) } \\
\text { Normal }(\leq 5.60 \mathrm{mmol} / \mathrm{L})\end{array}$ & $\begin{array}{l}116(39.2) \\
180(60.8)\end{array}$ \\
\hline $\begin{array}{l}\text { BMI } \\
\text { Underweight } \\
\text { Normal } \\
\text { Overweight }\end{array}$ & $\begin{array}{l}47(15.7) \\
192(64.2) \\
60(20.1)\end{array}$ \\
\hline $\begin{array}{l}\text { Exercise compliance } \\
\text { Yes } \\
\text { No }\end{array}$ & $\begin{array}{l}176(58.9) \\
123(41.1)\end{array}$ \\
\hline $\begin{array}{l}\text { BGL } \\
\text { Normal } \\
\text { High }\end{array}$ & $\begin{array}{l}187(62.7) \\
111(37.3)\end{array}$ \\
\hline
\end{tabular}

BGL: blood glucose level; BMI: body mass index; DM: diabetes mellitus.

variables was accessed using $95 \%$ Cls with a respective odds ratio (ORs) with the moderate-high adherence (not with low-adherence). $p<0.05$ was used as the cutoff point for statistical significance. Variables with $10 \%$ missing values were excluded from the analysis.

\section{Results}

\section{Demographic and clinical characteristics}

Out of the total approached patients, 398 initially agreed to participate in the study (response rate, $44.3 \%$ ), while only 299 of them completed the questionnaires. Age of respondents was $48.8 \pm 14.6$ years; $169(56.5 \%)$ participants were men, $270(90.3 \%)$ were married, 103 (34.5\%) were rural residents, and $68(22.7 \%)$ had income < 15,000 Pakistani rupee (PKR). There was a positive family history of DM for 204 (69.6\%) respondents, and 97 (32.4\%) had a positive family history of HTN (Table 1).

The mean duration of diabetes was $9.36 \pm 6.9$ years. About $15.4 \%$ were current smokers, and 176 (58.9\%) were compliant with exercise. Most respondents were in the normal BMI range (64.2\%); BGL was normal in $187(62.7 \%)$ participants, and 180 (60.8\%) had normal cholesterol levels (Table 2). 
Table 3. Questions related to patients adherence with medication*

\begin{tabular}{|c|c|c|}
\hline Questions & Yes (\%) & No (\%) \\
\hline Do you sometimes forget to take your medicine? & $33(11.0)$ & $266(89.0)$ \\
\hline $\begin{array}{l}\text { People sometimes miss taking medicine for reason other than forgetting. Thinking over the past } 2 \text { weeks, } \\
\text { were there any days when you are not taking your medicine? }\end{array}$ & $76(25.4)$ & $223(74.6)$ \\
\hline $\begin{array}{l}\text { Have you ever cut back or stop taking your medications without telling your doctor because you felt worse } \\
\text { when you took it? }\end{array}$ & $121(0.5)$ & $178(59.5)$ \\
\hline When you travel or leave home, do you sometimes forget to bring along your medication? & $44(14.7)$ & $255(85.3)$ \\
\hline Did you take your medications yesterday? & $271(90.6)$ & $28(9.4)$ \\
\hline When you feel like your diabetes is under control, do you sometimes stop taking your medications? & $78(34.1)$ & $221(73.9)$ \\
\hline $\begin{array}{l}\text { Taking medication every day is a real inconvenience for some people; do you ever feel hassled about sticking } \\
\text { to your treatment plan? }\end{array}$ & $34(11.4)$ & $265(88.6)$ \\
\hline $\begin{array}{l}\text { How often do you have difficulty remembering to take all your medicines? } \\
\text { Never/Rarely } 125(41.8) \\
\text { Once in a while } 35(11.7) \\
\text { Sometimes } 33(11.0) \\
\text { Usually } 38(12.8) \\
\text { All the time } 68 \text { (22.7) }\end{array}$ & & \\
\hline
\end{tabular}

Table 4. The different levels of adherence

\begin{tabular}{|l|c|c|}
\hline Adherence level & $\mathbf{n}$ & $\%$ \\
\hline Low adherence & 145 & 48.5 \\
\hline Moderate adherence & 69 & 23.1 \\
\hline High adherence & 85 & 28.4 \\
\hline
\end{tabular}

\section{Adherence rate with medications}

A total of $85(28.4 \%)$ were showing high adherence (MMAS-8 score 8), 69 (23.1\%) were showing moderate, and $145(48.5 \%)$ were showing low adherence. A total of $154(51.5 \%)$ patients were in the range of moderate to high adherence. The mean score for the compliance was $5.89 \pm 1.780$ (Tables 3 and 4).

The trend is showing the adherence decreased with age $(70.6 \%$ in age $<25$ vs. $44.2 \%$ in $>55$ years). The rural population tends to demonstrate less moderate-high adherence as compared to urban residents $(46.6 \%$ vs. $54.1 \%)$. Similarly, the increase in income and education showed an increasing trend in the moderate-high adherence of patients. About $42.6 \%$ of patients of income $<15,000 \mathrm{Pkr}$ showed moderate-high adherence, which is considerably less as compared to those having income more than 30,000 Pkr (59\%).

In terms of clinical characteristics influence on the adherence, it decreases with the increase in the duration of diabetes, those with the duration of more than 15 years have moderate-high adherence rate of $44.2 \%$ which is low as compared to moderate-high adherence rate shown by patients with duration $1-5$ years $(52.0 \%)$. Similarly, an increase in the number of comorbidities decreases the rate of adherence.

Patients who were helped by family members to take their medicine at the right time showed considerable high adherence $(60.8 \%$ vs. $46.7 \%)$. Similarly, those patients who had their own GM (57.0\%) were showing more adherence as compared to those without GM $(46.5 \%)$.

\section{Factor related to medication compliance}

The association between predictors and moderate-high adherence was investigated using univariate and multivariate analyses. Predictors showing an association with moderate-high adherence $(p \leq 0.25$ in the univariate analysis) were selected as candidate variables for multivariable logistic regression analysis. Relying on help from family members to take their medication correctly and fear that medicine will cause side effects significantly decrease compliance (Adjusted OR $[\mathrm{AOR}]=0.562 ; 95 \% \mathrm{Cl}=0.328,0.961$ and $\mathrm{AOR}=0.349$; $95 \% \mathrm{Cl}=0.192,0.635$, respectively). Both of these variables had significant associations with therapeutic moderate-high adherence $(p=0.035$ and 0.001 , respectively) (Tables 5 and 6). 
Table 5. Demographic characteristics and factors affecting compliance

\begin{tabular}{|c|c|c|c|c|c|c|}
\hline \multirow[t]{2}{*}{ Demographic characteristics } & \multirow[t]{2}{*}{ n (299) } & \multicolumn{5}{|c|}{ Medication adherence $\mathbf{n}(\%)$} \\
\hline & & $\begin{array}{l}\text { Low-adherent } \\
\text { (145) n (\%) }\end{array}$ & $\begin{array}{c}\text { Moderate-high } \\
\text { adherent (154) n (\%) }\end{array}$ & p value & AOR (95\% CI) & p value \\
\hline $\begin{array}{l}\text { Age } \\
\qquad 25 \\
26-40 \\
41-55 \\
\text { More than } 55\end{array}$ & $\begin{array}{c}17 \\
71 \\
116 \\
95\end{array}$ & $\begin{array}{l}5(29.4) \\
33(46.5) \\
55(47.4) \\
53(55.8)\end{array}$ & $\begin{array}{l}12(70.6) \\
39(53.5) \\
61(52.6) \\
42(44.2)\end{array}$ & 0.180 & $\begin{array}{c}1 \\
2.092(0.614,7.127) \\
1.149(0.580,2.274) \\
1.060(0.583,1.925)\end{array}$ & \\
\hline $\begin{array}{l}\text { Locality } \\
\text { Urban } \\
\text { Rural }\end{array}$ & $\begin{array}{l}196 \\
103\end{array}$ & $\begin{array}{l}90(45.9) \\
55(53.4)\end{array}$ & $\begin{array}{l}106(54.1) \\
48(46.6)\end{array}$ & 0.219 & $\begin{array}{c}1 \\
0.608(0.358,1.032)\end{array}$ & \\
\hline $\begin{array}{l}\text { Gender } \\
\text { Male } \\
\text { Female }\end{array}$ & $\begin{array}{l}169 \\
130\end{array}$ & $\begin{array}{l}83(49.1) \\
62(47.7)\end{array}$ & $\begin{array}{l}86(50.9) \\
68(52.3)\end{array}$ & 0.808 & & \\
\hline $\begin{array}{l}\text { BMI } \\
\text { Underweight } \\
\text { Normal } \\
\text { Overweight }\end{array}$ & $\begin{array}{c}47 \\
192 \\
60\end{array}$ & $\begin{array}{l}26(62.9) \\
95(49.5) \\
24(40.0)\end{array}$ & $\begin{array}{l}21(37.1) \\
97(50.5) \\
36(60.0)\end{array}$ & 0.261 & & \\
\hline $\begin{array}{l}\text { Monthly income } \\
<15,000 \\
15,000-30,000 \\
\text { More than } 30,000\end{array}$ & $\begin{array}{c}68 \\
134 \\
97\end{array}$ & $\begin{array}{l}39(57.4) \\
67(50.0) \\
39(40.2)\end{array}$ & $\begin{array}{l}29(42.6) \\
67(50.0) \\
58(59.8)\end{array}$ & 0.085 & $\begin{array}{c}1 \\
0.759(0.328,1.753) \\
1.098(0.468,2.574)\end{array}$ & \\
\hline $\begin{array}{l}\text { Marital status } \\
\text { Married } \\
\text { Single }\end{array}$ & $\begin{array}{c}270 \\
29\end{array}$ & $\begin{array}{l}134(49.6) \\
11(38.0)\end{array}$ & $\begin{array}{l}136(50.4) \\
18(62.0)\end{array}$ & 0.26 & & \\
\hline $\begin{array}{l}\text { Education } \\
\text { Less than primary } \\
\text { Primary-middle } \\
\text { Matric } \\
\text { Graduate }\end{array}$ & $\begin{array}{l}124 \\
65 \\
71 \\
39\end{array}$ & $\begin{array}{l}55(44.4) \\
39(60.0) \\
36(50.7) \\
15(38.5)\end{array}$ & $\begin{array}{l}69(55.6) \\
26(30.0) \\
35(49.3) \\
24(61.5)\end{array}$ & 0.111 & $\begin{array}{c}1 \\
0.914(0.405,2.060) \\
0.508(0.210,1.231) \\
0.698(0.296,1.644)\end{array}$ & \\
\hline $\begin{array}{l}\text { Family history of diabetes } \\
\text { Positive } \\
\text { Negative }\end{array}$ & $\begin{array}{c}208 \\
91\end{array}$ & $\begin{array}{l}106(51.0) \\
39(42.9)\end{array}$ & $\begin{array}{l}102(49.0) \\
52(57.1)\end{array}$ & 0.197 & $\begin{array}{c}1 \\
1.585(0.906,2.772)\end{array}$ & \\
\hline $\begin{array}{l}\text { Duration of diabetes (years) } \\
\begin{array}{l}1-5 \\
6-10 \\
10-15 \\
\text { More than } 15\end{array}\end{array}$ & $\begin{array}{c}100 \\
109 \\
47 \\
43\end{array}$ & $\begin{array}{l}48(48.0) \\
52(47.7) \\
21(44.6) \\
24(55.8)\end{array}$ & $\begin{array}{l}52(52.0) \\
57(52.3) \\
26(55.4 \\
19(44.2)\end{array}$ & 0.745 & & \\
\hline $\begin{array}{l}\text { Smoker } \\
\text { Yes } \\
\text { No }\end{array}$ & $\begin{array}{c}46 \\
253\end{array}$ & $\begin{array}{c}23(50.0) \\
122(48.2)\end{array}$ & $\begin{array}{c}23(50.0) \\
131(51.8)\end{array}$ & 0.824 & & \\
\hline $\begin{array}{l}\text { Co-morbidities } \\
\text { No } \\
1 \\
\text { More than } 1\end{array}$ & $\begin{array}{l}42 \\
40 \\
217\end{array}$ & $\begin{array}{c}18(42.9) \\
17(42.5) \\
110(50.7)\end{array}$ & $\begin{array}{c}24(57.1) \\
23(57.5) \\
107(49.3)\end{array}$ & 0.460 & & \\
\hline $\begin{array}{l}\text { Medications } \\
\text { Single therapy (OHG or insulin) } \\
\text { Combination therapy (OHG+ Insulin) }\end{array}$ & $\begin{array}{c}249 \\
50\end{array}$ & $\begin{array}{l}122(48.9) \\
23(46.0)\end{array}$ & $\begin{array}{l}127(51.1) \\
27(54.0)\end{array}$ & 0.699 & & \\
\hline $\begin{array}{l}\text { Polypharmacy } \\
\text { Yes } \\
\text { No }\end{array}$ & $\begin{array}{c}66 \\
233\end{array}$ & $\begin{array}{c}31(47.0) \\
114(49.0)\end{array}$ & $\begin{array}{c}35(53.0) \\
119(51.0)\end{array}$ & 0.779 & & \\
\hline $\begin{array}{l}\text { Do you take help of family member in } \\
\text { taking medication at the right time? } \\
\text { Yes } \\
\text { No }\end{array}$ & $\begin{array}{l}197 \\
102\end{array}$ & $\begin{array}{c}105(53.3) \\
40(39.2)\end{array}$ & $\begin{array}{l}92(46.7) \\
62(60.8)\end{array}$ & 0.000 & $\begin{array}{c}0.562(0.328,0.961) \\
1\end{array}$ & 0.035 \\
\hline
\end{tabular}


Table 5. Demographic characteristics and factors affecting compliance (Continued)

\begin{tabular}{|c|c|c|c|c|c|c|}
\hline \multirow[t]{2}{*}{ Demographic characteristics } & \multirow[t]{2}{*}{ n (299) } & \multicolumn{5}{|c|}{ Medication adherence n (\%) } \\
\hline & & $\begin{array}{l}\text { Low-adherent } \\
\text { (145) n (\%) }\end{array}$ & $\begin{array}{c}\text { Moderate-high } \\
\text { adherent (154) n (\%) }\end{array}$ & p value & AOR $(95 \%$ CI) & p value \\
\hline $\begin{array}{l}\text { Do you believe drug therapy cause } \\
\text { side effect to you? } \\
\text { Yes } \\
\text { No }\end{array}$ & $\begin{array}{c}74 \\
225\end{array}$ & $\begin{array}{l}51(68.9) \\
94(41.8)\end{array}$ & $\begin{array}{l}23(31.1) \\
131(58.2)\end{array}$ & 0.000 & $\begin{array}{c}0.349(0.192,0.635) \\
1\end{array}$ & 0.001 \\
\hline $\begin{array}{l}\text { Do you believe drug therapy is } \\
\text { beneficial to you? } \\
\text { Yes } \\
\text { No }\end{array}$ & $\begin{array}{c}269 \\
30\end{array}$ & $\begin{array}{l}129(48.0) \\
16(53.3)\end{array}$ & $\begin{array}{l}140(52.0) \\
14(46.7)\end{array}$ & 0.576 & & \\
\hline $\begin{array}{l}\text { Is your medicine affordable for you? } \\
\text { Yes } \\
\text { No }\end{array}$ & $\begin{array}{l}189 \\
110\end{array}$ & $\begin{array}{l}83(44.5) \\
71(64.6)\end{array}$ & $\begin{array}{l}105(55.5) \\
39(35.4)\end{array}$ & 0.066 & $\begin{array}{c}1.334(0.704,2.52) \\
1\end{array}$ & \\
\hline $\begin{array}{l}\text { Do you face any difficulty in getting } \\
\text { medicine? } \\
\text { Yes } \\
\text { No }\end{array}$ & $\begin{array}{c}95 \\
204\end{array}$ & $\begin{array}{l}46(48.4) \\
99(48.5)\end{array}$ & $\begin{array}{c}49(51.6) \\
105(51.5)\end{array}$ & 0.986 & & \\
\hline $\begin{array}{l}\text { Do you have your own glucometer? } \\
\text { Yes } \\
\text { No }\end{array}$ & $\begin{array}{l}142 \\
157\end{array}$ & $\begin{array}{l}61(43.0) \\
84(53.5)\end{array}$ & $\begin{array}{l}81(57.0) \\
73(46.5)\end{array}$ & 0.068 & $\begin{array}{c}1.820(0.911,3.635) \\
1\end{array}$ & \\
\hline $\begin{array}{l}\text { Do you sometimes feel confused with } \\
\text { the drug regimen? } \\
\text { Yes } \\
\text { No }\end{array}$ & $\begin{array}{l}148 \\
151\end{array}$ & $\begin{array}{l}83(56.0) \\
62(41.0)\end{array}$ & $\begin{array}{l}65(44.0) \\
89(59.0)\end{array}$ & 0.009 & $\begin{array}{c}0.760(0.444,1.3) \\
1\end{array}$ & \\
\hline
\end{tabular}

*Hosmer and Lemeshow is 0.847 for medication adherence.

\section{Correlation between BGL and medication adherence}

Possible relationships between BGL and medication adherence were demonstrated by the Spearman's rank-order correlation coefficient. The criteria for defining correlation were as follows: a correlation coefficient of 0-0.25 was considered weak correlation, 0.25-0.5 fair correlation, $0.5-0.75$ good correlation, and greater than 0.75 excellent correlation ${ }^{28}$. A weak, insignificant correlation was seen between medication adherence and BGL $(r=0.029, p=0.631)$.

\section{The prevalence of HTN and its risk factor in diabetics}

Among 299 patients, 133 (44.4\%) were diagnosed with HTN. The prevalence of HTN found to be highest among diabetics older than $55(61 \%)$ as compared to those aged $<25(17.7 \%)$. Urban residents show more prevalence $(47.0 \%)$ as compared to the rural population $(39.0 \%)$. Those who had a positive family history of HTN had a significantly high prevalence of HTN $(p<0.001)$. Taking into consideration the clinical outcomes increasing $\mathrm{BMI}$ increases the chances of being hypertensive, i.e., those who were overweight had a prevalence of $50.0 \%$, and those who were underweight had $36.2 \%$.

Predictors that were included in multivariate logistic regression ( $p \leq 0.25$ in bivariate analysis) were age, residence location, sex, monthly income, marital status, family history of HTN, duration of HTN, smoking, cholesterol level, and exercise compliance. Multivariate logistic regression analysis of HTN variables revealed that family history of HTN (AOR $=23.42 ; 95 \% \mathrm{Cl}=9.655$, 56.853; $p<0.001$ ), and abnormal cholesterol levels (AOR $=23.401 ; 95 \% \mathrm{Cl}=10.195,53.765 ; p=0.01$ ) were significantly associated with HTN (Table 6).

\section{Discussion}

Avoiding complications and achieving optimal health are the primary goals of DM management. This requires a combined effort from health-care officials and patients to improve adherence. Diabetes itself is a major influencing factor in the development of HTN. In this cross-sectional study, we measured the proportion of therapeutic adherence and prevalence of risk factors 
Table 6. The prevalence of risk factor of hypertension in diabetic patients

\begin{tabular}{|c|c|c|c|c|c|c|}
\hline Variables & $\mathbf{n}$ & $\begin{array}{c}\text { Absence of HTN } \\
n(\%)\end{array}$ & $\begin{array}{c}\text { Presence of HTN } \\
n(\%)\end{array}$ & p value & AOR $(95 \%$ CI) & p value \\
\hline $\begin{array}{l}\text { Age }(48.8 \pm 14.6) \\
\quad<25 \\
26-40 \\
41-55 \\
\text { More than } 55\end{array}$ & $\begin{array}{c}17 \\
71 \\
116 \\
95\end{array}$ & $\begin{array}{l}14(82.3) \\
54(76.0) \\
61(52.5) \\
37(39.0)\end{array}$ & $\begin{array}{l}3(17.7) \\
17(24.0) \\
55(48.5) \\
58(61.0)\end{array}$ & 0.1 & $\begin{array}{c}1 \\
0.604(0.086,3.103) \\
0.149(0.046,4.830) \\
0.676(0.246,1.35)\end{array}$ & \\
\hline $\begin{array}{c}\text { Locality } \\
\text { Urban } \\
\text { Rural }\end{array}$ & $\begin{array}{l}196 \\
103\end{array}$ & $\begin{array}{l}104(53.0) \\
62(61.0)\end{array}$ & $\begin{array}{l}92(47.0) \\
41(39.0)\end{array}$ & 0.238 & $\begin{array}{c}1 \\
0.654(0.291,1.467)\end{array}$ & \\
\hline $\begin{array}{l}\text { Gender } \\
\text { Male } \\
\text { Female }\end{array}$ & $\begin{array}{l}169 \\
130\end{array}$ & $\begin{array}{l}100(59.1) \\
66(50.7)\end{array}$ & $\begin{array}{l}69(40.9) \\
64(49.3)\end{array}$ & 0.147 & $\begin{array}{c}1 \\
1.757(0.795,3.883)\end{array}$ & \\
\hline $\begin{array}{l}\text { Monthly income (PKR) } \\
<15,000 \\
15,000-30,000 \\
\text { More than } 30,000\end{array}$ & $\begin{array}{c}68 \\
134 \\
97\end{array}$ & $\begin{array}{l}44(64.7) \\
73(54.4) \\
49(50.5)\end{array}$ & $\begin{array}{l}24(35.3) \\
61(44.6) \\
48(49.5)\end{array}$ & 0.186 & $\begin{array}{c}1 \\
0.779(0.276,2.203) \\
1.351(0.578,3.159)\end{array}$ & \\
\hline $\begin{array}{l}\text { Marital status } \\
\text { Married } \\
\text { Single }\end{array}$ & $\begin{array}{c}270 \\
29\end{array}$ & $\begin{array}{l}146(54.0) \\
20(69.0)\end{array}$ & $\begin{array}{c}124(46.0) \\
9(31.0)\end{array}$ & 0.125 & $\begin{array}{c}1 \\
0.727(0.207,2.548)\end{array}$ & \\
\hline $\begin{array}{l}\text { Education } \\
\text { Less than primary } \\
\text { Primary-Middle } \\
\text { Matric } \\
\text { Graduate }\end{array}$ & $\begin{array}{l}124 \\
65 \\
71 \\
39\end{array}$ & $\begin{array}{l}71(57.2) \\
31(47.7) \\
43(60.5) \\
21(53.8)\end{array}$ & $\begin{array}{l}53(42.8) \\
34(52.3) \\
28(39.5) \\
18(46.2)\end{array}$ & 0.468 & & \\
\hline $\begin{array}{l}\text { Family history of diabetes } \\
\text { Positive } \\
\text { Negative }\end{array}$ & $\begin{array}{c}208 \\
91\end{array}$ & $\begin{array}{l}116(55.7) \\
50(54.9)\end{array}$ & $\begin{array}{l}92(44.3) \\
41(45.1)\end{array}$ & 0.895 & & \\
\hline $\begin{array}{l}\text { Family history of HTN } \\
\text { Positive } \\
\text { Negative }\end{array}$ & $\begin{array}{c}97 \\
202\end{array}$ & $\begin{array}{c}15(15.5) \\
151(74.7)\end{array}$ & $\begin{array}{l}82(84.5) \\
51(25.3)\end{array}$ & $<0.001$ & $\begin{array}{c}23.42(9.655,56.853) \\
1\end{array}$ & $<0.001$ \\
\hline $\begin{array}{l}\text { Duration of diabetes (years) }(9.36 \pm 6.9) \\
1-5 \\
6-10 \\
11-15 \\
\text { More than } 15\end{array}$ & $\begin{array}{c}100 \\
109 \\
47 \\
43\end{array}$ & $\begin{array}{l}65(66.0) \\
64(58.7) \\
20(42.5) \\
17(39.5)\end{array}$ & $\begin{array}{l}35(34.0) \\
45(42.2) \\
27(57.5) \\
26(60.5)\end{array}$ & 0.008 & $\begin{array}{c}1 \\
0.643(0.135,1.635) \\
0.667(0.17,0.800) \\
1.032(0.244,2.017)\end{array}$ & \\
\hline $\begin{array}{l}\text { Smoker } \\
\text { Yes } \\
\text { No }\end{array}$ & $\begin{array}{c}46 \\
253\end{array}$ & $\begin{array}{c}22(47.8) \\
144(56.9)\end{array}$ & $\begin{array}{c}24(52.2) \\
109(43.1)\end{array}$ & 0.24 & $\begin{array}{c}1.532(0.537,4.366) \\
1\end{array}$ & \\
\hline $\begin{array}{l}\text { Total cholesterol } \\
\text { High } \\
\text { Normal }\end{array}$ & $\begin{array}{l}116 \\
180\end{array}$ & $\begin{array}{c}23(19.8) \\
142(78.8)\end{array}$ & $\begin{array}{l}93(80.2) \\
38(21.2)\end{array}$ & $<0.001$ & $\begin{array}{c}23.401(10.195,53.765) \\
1\end{array}$ & 0.001 \\
\hline $\begin{array}{l}\text { BMI } \\
\text { Underweight } \\
\text { Normal } \\
\text { Overweight }\end{array}$ & $\begin{array}{c}47 \\
192 \\
60\end{array}$ & $\begin{array}{c}30(63.8) \\
106(55.2) \\
30(50.0)\end{array}$ & $\begin{array}{l}17(36.2) \\
86(44.8) \\
30(50.0)\end{array}$ & 0.351 & & \\
\hline $\begin{array}{l}\text { Exercise compliance } \\
\text { Yes } \\
\text { No }\end{array}$ & $\begin{array}{l}176 \\
123\end{array}$ & $\begin{array}{l}104(59.0) \\
62(50.4)\end{array}$ & $\begin{array}{l}72(41.0) \\
61(49.6)\end{array}$ & 0.137 & $\begin{array}{c}0.781(0.359,1.7) \\
1\end{array}$ & \\
\hline $\begin{array}{l}\text { Diabetes medication adherence } \\
\text { Moderate-high } \\
\text { Low }\end{array}$ & $\begin{array}{l}154 \\
145\end{array}$ & $\begin{array}{l}89(57.8) \\
77(53.1)\end{array}$ & $\begin{array}{l}65(42.2) \\
68(46.9)\end{array}$ & 0.415 & & \\
\hline $\begin{array}{l}\text { BGL } \\
\quad \text { Normal } \\
\text { High }\end{array}$ & $\begin{array}{l}187 \\
111\end{array}$ & $\begin{array}{l}105(56.1) \\
60(54.0)\end{array}$ & $\begin{array}{l}82(43.9) \\
51(46.0)\end{array}$ & 0.725 & & \\
\hline
\end{tabular}

Hosmer and Lemeshow value was 0.634 for predictors of HTN.

AOR: adjusted odds ratio; BGL: blood glucose level; Cl: confidence interval; HTN: hypertension; PKR: Pakistani rupee. 
for HTN among DM patients in South Pakistan for the first time. The study population showed poor adherence to medication therapy. These results are in line with the previous studies conducted worldwide ${ }^{29}$, whereas the results of another study contradicted our results ${ }^{30}$. The difference in results may be attributed to different study periods, study locations, and study tools.

After adjustment for other factors in multivariable logistic regression, two factors were significantly associated with moderate-high adherence. DM patients who had family help or social support to take their medication correctly were less moderate-high adherent than those who do not have such support. The motivation for the self-management of diabetes is a pillar component in adherence. This attitude negates the need for dependence on primary care and thus strongly influences compliance. These results are in line with those of the previous studies ${ }^{31,32}$; however, some studies found positive associations between family or social support and adherence as such support reduces negative attitudes, improves motivation, and helps with remembering how to correctly take medication ${ }^{33}$.

Similarly, a study made in France demonstrated that the patients who were dependent on the family support showed significance high rate of good adherence than those who did not depend on the family support $(29.2$ vs. $2.4 \% p=0.0002)^{34}$. Those patients who believed that drug therapy caused them side effects were less likely to adhere to therapy. This factor can substantially affect patient compliance, as previous studies have showed ${ }^{35,36}$.

Adherence to treatment increased with age in our study. Patients aged $26-40$ years were showing more moderate-high adherence than those $<25$ years of age. This study result was in line with a report in the United States (USA) showing that age $<24$ years was a significant predictor of non-adherence to treatment ${ }^{37}$. Younger people are less concerned about the detrimental effects of DM and less interested in the importance of adherence. They are, therefore, more careless about their treatment. Rural populations demonstrated lower moderate-high adherence than urban ones (54.1\% vs. $46.6 \%)$. Rural areas lack basic health-care facilities and proper health education, and rural residents have low socioeconomic status, which all affect treatment adherence. Our population of income more than 30,000 PKR were $(59.8 \%)$ more likely to be moderate-high adherent than those with monthly incomes under 15,000 PKR (46.2\%). A study in France suggested that poor adherence was directly proportional to financial hardship. Those patients who do not have any financial burden were showing good adherence (20.3\%) than those who had some financial hardship (proper compliance $5.6 \%)(p<0.05)^{34}$. In this study, participants with higher education than matric $\left(10^{\text {th }}-11^{\text {th }}\right.$ high school) graduation were likely to be more adherent $(61.5 \%)$ as compared with those who had less than primary level educations (55.6\%). These results are in accordance with the previous studies ${ }^{38}$.

In our study, patients who could afford the cost of therapy were 1.334 times more likely to be adherent. The previous studies conducted in the US showed that the high cost of medication was a reason for lack of adherence, and a study in France associated poor adherence with the affordability of treatment ${ }^{34,39}$. A negative attitude or lack of motivation is another factor that hinders adherence. Patients in our study who felt confused or irritated by their drug regimen were less likely to be moderate-high adherent than those who did not. This effect was also seen in the previous studies showing that irritable temperament decreased adherence significantly ${ }^{30}$. A study in France showed that those patients who do not face difficulty in taking medications alone had a significantly high level of good adherence $^{34}$. Another study in China reported that patients with type $D$ personality were more likely to have poor adherence ${ }^{40}$. A total of $57 \%$ of patients who owned and used their own GM were moderate-high adherent which is a bit higher than those who did not own GM (46.5\%). The reason might be that proper glycemic monitoring keep patients motivated to stick with therapy. A study in Ethiopia found that patients who owned a GM were significantly less low-adherent, i.e., $15 \%$ those patients who had their own GM were low-adherent which is less as compared to those who do not own a GM $(42.8 \%$ non-adherent) $)^{41}$, and results of another study in the US demonstrated the positive response of self-monitoring blood glucose on sitagliptin treatment adherence ${ }^{42}$.

HTN is a silent killer that often accompanies DM. This was supported by the fact that $133(44.4 \%)$ participants with DM in our study were diagnosed with HTN; similar findings have been reported worldwide ${ }^{7}$. However, in one study conducted in India, the HTN prevalence was $<17.7 \%{ }^{43}$. After adjusting the other factors, such as age, family history of HTN, and cholesterol level, were significantly associated with the presence of HTN. Multivariate logistic regression analysis demonstrated that patients in the age group of 26-40 years were less likely to have HTN than those $<25$ years of age, although this contradicts previous reports. There is a significantly increased likelihood of developing HTN with increasing age ${ }^{43}$. The chances of having HTN among patients 
with a positive family history of HTN were 23.42 times greater than among those with no family history of HTN. There have been no studies correlating the relationship between family HTN history and HTN risk in populations with DM; however, a significantly increased risk of developing HTN with a positive family history has been reported previously in India ${ }^{43}$. HTN was 23.4 times more common among patients with high cholesterol levels than in those with a normal cholesterol level. The previous studies have not investigated cholesterol as a risk factor of HTN among diabetes patients; however, studies conducted among patients with HIV have demonstrated high cholesterol levels to be a risk factor for the development of $\mathrm{HTN}^{44}$, with similar results reported in a US study ${ }^{45}$.

\section{Limitations}

First, the response rate of the population in our study was very low due to the hasty environment and disinterest in the activity. Second, the comparisons of adherence were made against the BGL which is not the parameter used to represent the disease management outcome. HBA1c comparisons instead provide a proper understanding of one's disease management. However, resources did not allow calculating the HBA1c measurements, so the previous comparisons were made. Third, the selection process was based on systemic random sampling which cannot give the actual representativeness of the sample. However, this problem could be reduced by the selection of a large sample size, which was difficult due to a low response rate. Finally, recall biases may be present due to the face to face interview. This limitation was minimized by cross-checking as many variables as we could.

\section{Conclusions}

Dependence on the family for diabetes management and a belief that drug treatment is causing side effects is risk factors for moderate-high adherence with DM treatment. This situation can be rectified by improving health education and proper counseling. Health-care providers should focus on educating, motivating, and improving the skills of DM patients, which will ultimately improve their adherence. The prevalence of risk factors for HTN was high among DM patients. These must be monitored regularly and controlled to prevent HTN.

\section{Ethics approval and informed consent}

The study followed the tenets of the Declaration of Helsinki and was approved by the Ethics Committee for Medical Research of Xi'an Jiaotong University in Shaanxi, China (Ref \# DIAB-16-19). This study was further approved by the Ethics Committee of Islamia University Bahawalpur. Written approval was also obtained from the medical superintendent of the respective study hospitals and physicians at private clinics. Willing literate patients were then asked to sign the consent form approved by the Ethics Committee. Illiterate patients were clarified about the contents of the written consent form, and the data collector asked permission to sign the consent form on their behalf.

\section{Acknowledgment}

We acknowledge the participation of all the respondents. We do also acknowledge the help of data collectors. The MMAS (8-item) content, name, and trademarks are protected by US copyright and trademark laws. Permission for the use of the scale and its coding is required. A license agreement is available from Donald E. Morisky, ScD, ScM, MSPH, $14725 \mathrm{NE} 20^{\text {th }}$ St. Bellevue, WA 98007, USA; dmorisky@gmail.com.

\section{Conflicts of interest}

The authors disclose no conflicts of interest.

\section{Funding}

This work was funded by the "Young Talent Support Program" of Xian Jiaotong University and China Medical Board (Grant No. 16-262).

\section{Ethical disclosures}

Protection of human and animal subjects. The authors declare that no experiments were performed on humans or animals for this study.

Confidentiality of data. The authors declare that they have followed the protocols of their work center on the publication of patient data.

Right to privacy and informed consent. The authors declare that no patient data appear in this article. 


\section{References}

1. Shrivastava SR, Shrivastava PS, Ramasamy J. Role of self-care in management of diabetes mellitus. J Diabetes Metab Disord. 2013;12:14.

2. Diabetes in Pakistan; 2015. Available from: http://www.idf.org/membership/mena/pakistan. [Last accessed on 2020 Feb 16]

3. Narayan KM, Gregg EW, Fagot-Campagna A, Engelgau MM, Vinicor F. Diabetes a common, growing, serious, costly, and potentially preventable public health problem. Diabetes Res Clin Pract. 2000;50 Suppl 2:S77-84.

4. American College of Preventive Medicine. Medicine ARftACoP medication adherence time tool: improving health outcomes. In: Medication Adherence Clinical Reference. Washington, DC: American College of Preventive Medicine; 2011. [cited 2016 Nov 22]. Available from: http:// www.acpm.org/?Adherence.

5. Abebe SM, Berhane Y, Worku A. Barriers to diabetes medication adherence in North West Ethiopia. Springerplus. 2014:3:195.

6. Tricco AC, Ivers NM, Grimshaw JM, Moher D, Turner L, Galipeau J, et al. Effectiveness of quality improvement strategies on the management of diabetes: a systematic review and meta-analysis. Lancet. 2012;379:2252-61.

7. Epstein M, Sowers JR. Diabetes mellitus and hypertension. Hypertension. 1992;19:403-18.

8. Sackett DL, Haynes RB, Gibson ES, Hackett BC, Taylor DW, Roberts RS, et al. Randomised clinical trial of strategies for improving medication compliance in primary hypertension. Lancet. 1975;1:1205-7.

9. Murphy J, Coster G. Issues in patient compliance. Drugs. 1997;54: 797-800.

10. Norris SL, Lau J, Smith SJ, Schmid CH, Engelgau MM. Self-management education for adults with Type 2 diabetes: a meta-analysis of the effect on glycemic control. Diabetes Care. 2002;25:1159-71.

11. Salam MA, Siddiqui AF. Socio-demographic determinants of compliance among Type 2 diabetic patients in Abha, Saudi Arabia. J Clin Diagn Res 2013;7:2810-3

12. Jin J, Sklar GE, Min Sen Oh V, Chuen Li S. Factors affecting therapeutic compliance: a review from the patient's perspective. Ther Clin Risk Manag 2008;4:269-86.

13. National Cholesterol Education Program (NCEP) Expert Panel on Detection, Evaluation, and Treatment of High Blood Cholesterol in Adults (Adult Treatment Panel III). Third report of the national cholesterol education program (NCEP) expert panel on detection, evaluation, and treatment of high blood cholesterol in adults (Adult Treatment Panel III) final report. Circulation. 2002;106:3143-421.

14. Peyrot M, Rubin RR. Modeling the effect of diabetes education on glycemic control. Diabetes Educ. 1994;20:143-8.

15. Ciechanowski PS, Katon WJ, Russo JE, Walker EA. The patient-provider relationship: Attachment theory and adherence to treatment in diabetes. Am J Psychiatry. 2001;158:29-35.

16. Nazir SU, Hassali MA, Saleem F, Bashir S, Aljadhey H. Disease related knowledge, medication adherence and glycaemic control among patients with Type 2 diabetes mellitus in Pakistan. Prim Care Diabetes. 2016;10:136-41.

17. Gillan $A H$, Ahmed $A B$, Bashir S, Ibrahim MI, Fang Y. Associated factors with diet and exercise compliance and smoking habits in diabetic patients of Punjab Pakistan. Med Univ. 2018;20:4-12.

18. Morisky DE, Ang A, Krousel-Wood M, Ward HJ. Predictive validity of a medication adherence measure in an outpatient setting. J Clin Hypertens (Greenwich). 2008;10:348-54.

19. Berlowitz DR, Foy CG, Kazis LE, Bolin LP, Conroy MB, Fitzpatrick P, et al. Effect of intensive blood-pressure treatment on patient-reported outcomes. N Engl J Med. 2017;377:733-44.

20. Morisky DE, DiMatteo MR. Improving the measurement of self-reported medication nonadherence: response to authors. J Clin Epidemiol. 2011;64:255-7

21. Saleem F, Hassali M, Akmal S, Morisky DE, Muhammad A, Harith KH, et al. Translation and validation study of Morisky Medication Adherence Scale (MMAS): the Urdu version for facilitating person-centered healthcare in Pakistan. Int J Person Centered Med. 2012;2:384-90.

22. Raum E, Krämer HU, Rüter G, Rothenbacher D, Rosemann T, Szecsenyi J, et al. Medication non-adherence and poor glycaemic control in patients with Type 2 diabetes mellitus. Diabetes Res Clin Pract. 2012;97:377-84.
23. Kang CD, Tsang PP, Li WT, Wang HH, Liu KQ, Griffiths SM, et al. Determinants of medication adherence and blood pressure control among hypertensive patients in Hong Kong: a cross-sectional study. Int J Cardiol. 2015;182:250-7.

24. Body Mass Index. Available from: http://www.euro.who.int/en/health-topics/disease-prevention/nutrition/a-healthy-lifestyle/body-mass-indexbmi. [Last accessed on 2020 Nov 26].

25. What is a Normal Blood Sugar Level? Available from: https://www.diabetesselfmanagement.com/blog/what-is-a-normal-blood-sugar-level. [last accessed on 2019 Nov 26]

26. Arega Sadore A, Abebe Gebretsadik L, Aman Hussen M. Compliance with iron-folate supplement and associated factors among antenatal care attendant mothers in Misha district, South Ethiopia: community based cross-sectional study. J Environ Public Health. 2015;2015:781973.

27. Burgess S, CRP CHD Genetics Collaboration. Identifying the odds ratio estimated by a two-stage instrumental variable analysis with a logistic regression model. Stat Med. 2013;32:4726-47.

28. UI Haq N, Hassali MA, Shafie AA, Saleem F, Farooqui M, Aljadhey $H$ A cross sectional assessment of knowledge, attitude and practice towards Hepatitis B among healthy population of Quetta, Pakistan. BMC Public Health. 2012;12:692.

29. Cramer JA, Benedict A, Muszbek N, Keskinaslan A, Khan ZM. The significance of compliance and persistence in the treatment of diabe tes, hypertension and dyslipidaemia: a review. Int J Clin Pract. 2008:62:76-87.

30. Shamsi A, Khodaifar F, Arzaghi SM, Sarvghadi F, Ghazi A. Is there any relationship between medication compliance and affective temperaments in patients with Type 2 diabetes? J Diabetes Metab Disord. 2014;13:96.

31. Gherman A, Schnur J, Montgomery G, Sassu R, Veresiu I, David D. How are adherent people more likely to think? A meta-analysis of health beliefs and diabetes self-care. Diabetes Educ. 2011;37:392-408.

32. Rosland AM, Kieffer E, Israel B, Cofield M, Palmisano G, Sinco B, et al. When is social support important? The association of family support and professional support with specific diabetes self-management behaviors. J Gen Intern Med. 2008;23:1992-9.

33. DiMatteo MR. Social support and patient adherence to medical treatment: a meta-analysis. Health Psychol. 2004;23:207-18.

34. Tiv M, Viel JF, Mauny F, Eschwège E, Weill $A$, Fournier $C$, et al. Medication adherence in Type 2 diabetes: the ENTRED study 2007, a French population-based study. PLoS One. 2012;7:e32412.

35. Farmer A, Kinmonth AL, Sutton S. Measuring beliefs about taking hypoglycaemic medication among people with Type 2 diabetes. Diabet Med. 2006;23:265-70.

36. Grant RW, Devita NG, Singer DE, Meigs JB. Polypharmacy and medication adherence in patients with Type 2 diabetes. Diabetes Care. 2003;26:1408-12.

37. Hertz RP, Unger AN, Lustik MB. Adherence with pharmacotherapy for Type 2 diabetes: a retrospective cohort study of adults with employer-sponsored health insurance. Clin Ther. 2005;27:1064-73.

38. Spikmans FJ, Brug J, Doven MM, Kruizenga HM, Hofsteenge GH, van Bokhorst-van der Schueren MA. Why do diabetic patients not attend appointments with their dietitian? J Hum Nutr Diet. 2003;16:151-8.

39. Odegard PS, Gray SL. Barriers to medication adherence in poorly controlled diabetes mellitus. Diabetes Educ. 2008;34:692-7.

40. Li X, Zhang S, Xu H, Tang X, Zhou H, Yuan J, et al. Type d personality predicts poor medication adherence in chinese patients with Type 2 diabetes mellitus: a six-month follow-up study. PLoS One. 2016;11:e0146892.

41. Kassahun A, Gashe F, Mulisa E, Rike WA. Nonadherence and factors affecting adherence of diabetic patients to anti-diabetic medication in Assela General Hospital, Oromia Region, Ethiopia. J Pharm Bioallied Sci. 2016;8:124-9.

42. Nigam S, Virdi NS, Daskiran M, Kozma CM, Paris A, Dickson WM. Association between sitagliptin adherence and self-monitoring of blood glucose. J Diabetes Sci Technol. 2012;6:555-62.

43. Panesar S, Chaturvedi S, Saini NK, Avasthi R, Singh A. Prevalence and predictors of hypertension among residents aged 20-59 years of a slum-resettlement colony in Delhi, India. WHO South East Asia J Public Health. 2013;2:83-7.

44. Thiébaut R, El-Sadr WM, Friis-Møller N, Rickenbach M, Reiss P, Monforte AD, et al. Predictors of hypertension and changes of blood pressure in HIV-infected patients. Antivir Ther. 2005;10:811-23.

45. Men with High Total Cholesterol are much more Likely to Develop High Blood Pressure Thanmen with Low Total Cholesterol. Available from: http:// www.newsmedical.net/news/2005/12/15/15003.aspx. [Last accessed on 2016 Nov 03] 